\title{
WHAT IS A MATTER PARTICLE?
}

\author{
TSAN UNG CHAN \\ Laboratoire de Physique Subatomique et de Cosmologie \\ IN2P3-CNRS et Université Joseph Fourier \\ 53 Avenue des Martyrs, F38026 Grenoble Cedex France \\ tsan@1psc.in2p3.fr
}

Positive baryon number $(\mathrm{A}>0)$ and positive lepton number $(\mathrm{L}>0)$ characterize matter particles while negative baryon number and negative lepton number characterize antimatter particles. Matter particles and antimatter particles belong to two distinct classes of particles. Matter neutral particles are particles characterized by both zero baryon number and zero lepton number. This third class of particles includes mesons formed by a quark and an antiquark (a pair of matter particle and antimatter particle) and bosons which are messengers of known interactions (photons for electromagnetism, $\mathrm{W}$ and $\mathrm{Z}$ for Weak Interaction, gluons for Strong Interaction). The antiparticle of a matter particle belongs to the class of antimatter particles, the antiparticle of an antimatter particle belongs to the class of matter particles. The antiparticle of a matter neutral particle belongs to the same class of matter neutral particles. A truly neutral particle is a particle identical with its antiparticle: it belongs necessarily to the class of matter neutral particles. All known interactions of the Standard Model do conserve baryon number and lepton number: it reflects in fact that matter cannot be created or destroyed via a reaction governed by these interactions. Conservation of baryon and lepton number parallels conservation of atoms in chemistry: the number of atoms of a particular species in the reactants must equal the number of those atoms in the products. These laws of conservation valid for interaction involving matter particles are indeed valid for any particles (matter particles characterized by positive numbers, antimatter particles characterized by negative numbers, and matter neutral particles characterized by zero). Interactions of the Standard Model which conserve both matter and Q in microscopic level can thus not explain the observed asymmetry of our Universe. Strong Interaction was introduced to explain the stability of nuclei: there must exist a powerful force to compensate electromagnetic force which tends to cause protons to fly apart. Weak Interaction with laws of conservation different from Electromagnetism and Strong Interaction was postulated to explain beta decay. Likely, our observed material and neutral universe would be footprint of the existence of another interaction that did conserve $\mathrm{Q}$ but did not conserve matter. 


\section{Introduction}

The substance of Earth is made up, by definition, of matter particles (or simply particles when there is no confusion): electrons, protons and neutrons. Combinations of these particles form atoms. There are more than 100 different kinds of atoms, each kind constituting a unique chemical element associated to the number of protons in its nuclei. A combination of atoms forms a molecule. Atoms and/or molecules can join together to form a compound. Any macroscopic material object can be considered to be at last formed by these three fundamental bricks. Ordinary matter is made up of protons, neutrons and electrons. Indeed we know that our Universe is a matter Universe in the sense that it is essentially made up of electrons, protons and neutrons.

\section{Conservation of matter in chemical and nuclear reactions}

Chemical reactions (governed by electromagnetic interaction) are represented by chemical equations which basically are connected to the conservation of matter under the following statement: The number of atoms of a particular species in the reactants must equal the number of those atoms in the products (stoichiometric balance). A chemical equation must be balanced because atoms are neither created nor destroyed. When the equation is balanced the identities of the reactants and the products remain unchanged. An example of chemical equation is : $\mathrm{C}+\mathrm{O}_{2} \rightarrow \mathrm{CO}_{2}$

There is a counting relation between positive integer numbers of the two members. The direct corollary of the conservation of atoms is the conservation of mass if one assumes that a specific atom has a defined mass whatever its combination with other atoms is. In the above example, $\mathrm{C}$ atom has the same mass as the $\mathrm{C}$ atom in $\mathrm{CO}_{2}$ and $\mathrm{O}$ atom has the same mass as the $\mathrm{O}$ atom in $\mathrm{CO}_{2}$. Conservation of matter under the form of conservation of mass was established by Lavoisier from his very precise measurements with a balance. Another important aspect of a chemical reaction is its $Q$-value.

For example, $\mathrm{C}+\mathrm{O}_{2} \rightarrow \mathrm{CO}_{2} \quad$ is an exothermic reaction where $Q$-value is the energy obtained in the form of heat $\left(Q\right.$-value $=393 \mathrm{~kJ} /$ mole or $65.2510^{-20} \mathrm{~J} /$ molecule or 4.07 $\mathrm{eV} /$ molecule)

With hindsight, since mass is only one form of energy, conservation of mass has in principle to be subsumed by the general law of conservation of energy, a mass $m$ having an energy $\mathrm{E}=\mathrm{mc}^{2}$. Generally the $Q$-value of reaction is different from zero, it thus implies that strictly speaking, the sum of the mass of the reactants is not equal to the sum of the mass of the products if $Q$-value is not zero, but in fact the difference of mass corresponding to $Q$ value is so tiny that it is not detectable. Thus, in chemistry, Lavoisier law stating that in a 
chemical reaction matter (revealed and represented by mass) cannot be created nor destroyed, but can only be changed from one form to another is still valid and experimentally verified to great accuracy. There is as much mass or weight present before and after a reaction. The substances have only changed their forms.

Understanding of a nuclear reaction (governed by strong interaction), for example,

$$
{ }^{7} \mathrm{Li}+\mathrm{p} \rightarrow{ }^{4} \mathrm{He}+{ }^{4} \mathrm{He}
$$

is based on similar counting relation, proton and neutron playing here the role of elements in chemical reaction. Conservation of matter implies here the conservation of the number of protons and the number of neutrons (proton and neutron are neither created nor destroyed). If we define in a nucleus the number of protons as $\mathrm{Z}$ and the number of neutrons as $\mathrm{N}$, then the mass number $\mathrm{A}=\mathrm{Z}+\mathrm{N}$ is the sum of protons and neutrons which are collectively named nucleons. $\mathrm{A}$ is the number of nucleons. Conservation of $\mathrm{Z}$ and $\mathrm{N}$ is equivalent to conservation of A and electric charge Q. Reactions governed by Strong Interaction involving only protons and neutrons conserve $\mathrm{A}$ and $\mathrm{Q}$. We remark that conservation of elements in chemical reaction implies automatically conservation of the number of protons, the number of neutrons and the number of electrons involved in a chemical reaction. The number of atoms, A, Z and $\mathrm{N}$ are naturally positive integer numbers. It is then clear that if we have 0 particle (element) in the entrance channel we cannot obtain particles (elements) in the exit channel (creation of matter) or the addition of two particles (elements) cannot give 0 particle (element) in the exit channel (destruction of matter). As in arithmetic the sum of two positive numbers cannot be 0 .

The $Q$-value of the reaction (corresponding to the difference of mass between the entrance channel and the exit channel) ${ }^{7} \mathrm{Li}+\mathrm{p} \rightarrow{ }^{4} \mathrm{He}+{ }^{4} \mathrm{He}$ is $17.3 \mathrm{MeV}$ and is much more important (many orders of magnitude) than in the chemical case (electron, proton and neutron are neither created nor destroyed). This reaction with large $Q$-value proved clearly the transformation of mass into energy and mass is clearly not conserved in this reaction.

\section{Concept of baryon number $A$ and lepton number $L$}

Later, it was realized that to explain strange particles, it was necessary to replace the conservation of nucleons by conservation of baryon number which characterizes not only nucleons but also heavy particles other than nucleons. "Experiment tells us that we can no longer talk about conservation of nucleons only but that by heavy particles one has to understand the totality of at least nucleons and $\mathrm{V}_{1}$-particles. Without prejudging on the actual nature of the relationship between the $\mathrm{V}_{1}$ and the nucleons it seems practical to have a collective name for these particles and other which possibly may still be discovered and which may also to be taken along in the conservation principle just mentioned. It is proposed 
to use the fitting name "baryon" for this purpose" [1]. Nucleons (proton or neutron) have naturally baryon number $A=1$. But indeed, $A=1$ characterizes any particle formed by three quarks whatever their flavours are. Baryons are fermions. Another class of particles called collectively mesons is observed in reactions governed by Strong Interaction. They are bosons characterized by $\mathrm{A}=0$. Mesons are indeed formed by a quark and an antiquark. Baryons and mesons which are composite particles formed by coloured quarks are sensitive to Strong Interaction even if their colours are globally neutral: they do feel residual interaction. On the contrary electron and neutrino belong to another distinct class of elementary particles which are truly colourless and thus insensitive to Strong Interaction. By analogy with A to characterize particles sensitive to Strong Interaction and Weak Interaction, lepton number L characterizes particles insensitive to Strong Interaction but sensitive to Weak Interaction: both electron and neutrino have $\mathrm{L}=1$. Neutrino is electrically neutral so it is also insensitive to electromagnetic force. It is clear that hadrons have $\mathrm{L}=0$ and leptons have $\mathrm{A}=0$. In a reaction governed by Strong Interaction, we observe only hadrons.

When charged mesons $(\mathrm{A}=0)$ are involved, conservation of $\mathrm{Z}$ and $\mathrm{N}$ is not valid while conservation of $\mathrm{A}$ and $\mathrm{Q}$ is still valid and explains naturally the occurrence of reactions governed by Strong Interaction like $p+p \rightarrow p+n+\pi^{+}$

On the contrary the non observation of $p+p \rightarrow p+p+n$ reflects that this reaction is strictly forbidden due to the non-conservation of $\mathrm{A}$, e.g. the non conservation of matter (number of baryons) in the realm of hadrons. Conservation of $A$ and $Q$ subsumes conservation of $Z$ and $N$ and more generally conservation of $\mathrm{A}, \mathrm{L}$ and $\mathrm{Q}$ subsumes conservation of the number of protons, the number of neutrons and the number of electrons in a chemical reaction. We remark also that the number of fermions should be conserved, while it is not necessary to conserve the number of mesons which are bosons because mesons have $\mathrm{A}=0$. We define the Matter number $M$ as the sum of $A$ and $L: M=A+L . M$ is positive for any matter particle. Conservation of matter means conservation of $\mathrm{A}$ and conservation of $\mathrm{L}$ in reactions where only matter particles $(\mathrm{A}>0)$ and particles with $\mathrm{A}=0$ are involved.

\section{First concept of antiparticle: antielectron was defined in connection to matter particle electron}

In the 1930s, the existence of antielectron was anticipated by the equation of Dirac, and the unexpected observation of positrons by Anderson at the same epoch validated the concept of the antiparticle. At that time, matter particles: proton, neutron, electron (fermions) were experimentally observed while neutrino was still a postulated particle $(\operatorname{spin}=1 / 2, Q=0)$ invented by Pauli to account for the continuous spectrum of electron in beta decay. Its actual 
existence as a real particle was clearly demonstrated only in 1953. Electron, proton and neutron are naturally considered to be particles, particles meaning in fact matter particles. Antielectron is the first detected antiparticle, antiparticle meaning antimatter particle. We note however that antiparticles as well as their counterparts particles, are indeed real particles in the sense that they are experimentally detectable: for example electron and positron are both deviated in a magnetic field but their curvatures are opposite due to opposite electric charges. At that time, only mass, spin and charge were well known characteristics of a particle. Antielectron should have same mass, same spin but opposite charge to electron. Antielectron and electron are distinct by their charges. Likely, proton and antiproton differ also by their charges. Antiparticles (since electron and proton are matter particles, positron and antiproton are naturally considered as antimatter particles) were thus revealed by opposite charge to the charge of well known electron and proton. An antiparticle was thus defined as a particle with the same mass, same spin but opposite charge to the corresponding well known matter particle. We remark nevertheless that the sign of $\mathrm{Q}$ is not associated to matter particle or antimatter particle. The charge of proton is 1 while the charge of electron is -1 but proton and electron are both matter particles. The electric charge $\mathrm{Q}$ of proton, neutron and electron which are all matter particles are respectively 1,0 and -1 . Thus the value of $Q$ alone can not reveal if a particle is a matter particle or an antimatter particle. The reality of antimatter particles is established experimentally by detecting them with usual techniques. Observation of creation (or annihilation) of a pair of matter particle and antimatter particle is now an everyday fact in particle physics.

\section{Charge is not the only difference between matter particle and antimatter particle}

Initially equation of Dirac concerned electron, a charged particle with spin $1 / 2$ (valid for proton which is also a charged particle with spin $1 / 2$ ). Its antiparticle was defined as a particle with the same spin and the same mass as the considered particle but with opposite charge. This original definition limited to the electric charge $Q$, the only well known algebraic property at that time, which played a central role in the identification of first charged antiparticle via its behaviour in electromagnetic field, appeared soon incomplete, for it raised immediately the problem of neutral fermions (matter particles) such as neutron and neutrino which are the neutral matter particles. Is a neutral matter particle identical to its antiparticle since they are not distinct by their charges or is it possible that it is nevertheless distinct from its antiparticle by other yet unknown algebraic properties? In 1937, Majorana [2] posed explicitly the following question: Are antineutron and antineutrino identical to neutron and neutrino since $\mathrm{Q}=0$ or are they different from their antiparticles as their charged matter 
siblings such as proton antiproton, electron antielectron? He had indeed the intuition that Q which is the most evident difference between particle and antiparticle is not sufficient to characterize a given particle whose identity card should contain other yet unknown arithmetical and especially algebraic properties. In other words the nature of neutral particle is not decidable if $\mathrm{Q}=0$ is the only known algebraic property. But he did not suggest criteria or experiments to decide between his new theory (postulating that neutron and neutrino are their own antiparticles) and a simple extension of the Dirac equation to distinct neutral particles. It is obvious that if neutral matter particle and its neutral antimatter particle are really different then the ambiguity could and should be lifted by at least one other non null algebraic property. Racah [3] pointed out immediately that magnetic moment, an algebraic quantity known in classical physics and in quantum physics could be used to lift this ambiguity. Since neutron has clearly non null magnetic moment, neutron and antineutron are distinct. Proton, neutron being characterized by $\mathrm{A}=1$, it is natural to characterize antiproton and antineutron by $A=-1$. Likewise electron is characterized by $L=1$ and antielectron is characterized by $L=-1$. The concept of antiparticles doubles thus the number of particles, each matter particle having a distinct corresponding antimatter particle. This extension is very similar to the extension of the notion of positive numbers to negative numbers [4]. A matter particle is thus necessarily different from its corresponding antimatter particle whatever the other algebraic properties are. $\mathrm{M}=\mathrm{A}+\mathrm{L}$ is positive for matter particle and negative for antimatter particle. But there is a great difference between numbers and particles. There is only one number for a given value. A number and its opposite number are clearly different. The only exception is 0 which is the only number equal to its opposite number. On the contrary, there are many particles with the same A, for example all baryons are characterized by $A=1$ and they are indeed distinct by other properties (arithmetic properties like mass, half-live or algebraic properties like magnetic dipole moment, electric dipole moment, baryon number, flavour numbers). We remark that pentaquark consisting of four quarks and one antiquark, if it exists, is also characterized by $\mathrm{A}=1$ and thus should be considered as a matter particle. We point out that in all known interactions of the Standard Model namely electromagnetism, Strong Interaction and Weak Iinteraction, conservation of matter (in the form of conservation of $\mathrm{A}$ and conservation of $\mathrm{L}$ ) which is valid when involved particles are all matter particles remains still valid in the general case where matter or/and antimatter particles are involved provided one takes into account their algebraic values. Protons, neutrons and electrons are elementary bricks of ordinary matter of everyday life while antiprotons, antineutrons and antielectrons are elementary bricks of antimatter. They belong to two completely distinct groups $(\mathrm{M}>0$ for 
matter particles and $\mathrm{M}<0$ for antimatter particles). It turns out that all other subsequently discovered algebraic properties (for example flavours) of a given matter particle and its antiparticle are always opposite. In consequence, any non null algebraic property (magnetic dipole moment, electric dipole moment, baryon number, lepton number, flavour number ...) of a neutral particle is sufficient to infer that it is different from its antiparticle even if all other known algebraic properties are null. $\mathrm{M}>0(\mathrm{~A}>0$ or $\mathrm{L}>0)$ is necessarily associated to matter particles (analogous to positive numbers), $\mathrm{M}<0(\mathrm{~A}<0$ or $\mathrm{L}<0)$ is necessarily associated to antimatter particles (analogous to negative numbers) while the sign of other algebraic properties of a particle does not allow to state that it is a matter particle or an antimatter particle. An immediate consequence is that a matter particle ( $\mathrm{A}>0$ or $\mathrm{L}>0)$ cannot be identical with its antiparticle which should be an antimatter particle $(\mathrm{A}<0$ or $\mathrm{L}<0)$.

\section{Relationship between $Q, A, L$ and flavours}

A sacred dogma in physics states that $\mathrm{Q}$ is always conserved. We remark that to this day, all observed reactions do conserve $\mathrm{Q}$ without any exception. Conservation of $\mathrm{Q}$ is thus verified experimentally again and again. Even hypothetical force responsible of the creation of our Universe which began in a super dense initial state of perfect symmetry without matter particles and without antimatter particles, since our Universe is clearly material and electrically neutral, did conserve $\mathrm{Q}$ and this conservation should be considered as an empirical fact and not just as a theoretical dogma. However, if electromagnetism depends only on the value of $\mathrm{Q}, \mathrm{Q}$ is indeed intimately related to other additive quantum numbers via generalized Gell-Mann and Nishijima formula written in the following form [5, 6]:

$$
\begin{aligned}
\mathrm{Q} & =\frac{\mathrm{THF}}{2}+\frac{\mathrm{A}}{2}+\frac{\mathrm{TLF}}{2}-\frac{\mathrm{L}}{2} \\
\text { or } \quad \mathrm{Q} & =\frac{\mathrm{TF}}{2}+\frac{\mathrm{A}}{2}-\frac{\mathrm{L}}{2}
\end{aligned}
$$

with $\mathrm{TF}=\mathrm{THF}+\mathrm{TLF}$

where $\mathrm{A}$ is the baryon number, $\mathrm{L}$ is the lepton number, THF is the total hadronic flavour, TLF is the total leptonic flavour and TF the total flavour. Due to this relation, conservation of $\mathrm{Q}$ means not only conservation of the value of $\mathrm{Q}$ but conservation of other quantities: Strong interaction and electromagnetism require that $\mathrm{A}, \mathrm{L}$ and all individual flavours are conserved while Weak Interaction requires only the conservation of $\mathrm{A}, \mathrm{L}$ and $\mathrm{TF}$.

\section{Significance of positive, negative and null values of $A$ or/and $L$}

Numbers are positive, negative or zero. Any positive number is different from its opposite number by its sign. 0 is neither positive nor negative (both positive and negative) and 
is the only number equal to its opposite. In the realm of particles it is evident that any matter particle is distinct from its corresponding antiparticle since they differ at least by the sign of A or $\mathrm{L}$, as any positive number is different from its opposite number which is negative. We note however that the concept of particle (and antiparticle) is not limited to matter particle and antimatter particle. There is a whole class of matter neutral particles characterized by $\mathrm{A}=0$ and $\mathrm{L}=0$ which are neither matter particle nor antimatter particle (bosons, messengers of forces like photon, $\mathrm{W}, \mathrm{Z}$ and gluons) or both matter particle and antimatter particle (mesons which are composite particles formed by a quark and an antiquark). Mesons and baryons are collectively called hadrons which are colour neutral combination of quarks. Messengers are on the contrary elementary and intrinsically matter neutral particles. Particles with $A=0$ and $\mathrm{L}=0$ (neutral matter particles group) belong to a third group of particles distinct from matter particles group $(A>0$ or $\mathrm{L}>0)$ or antimatter particles group $(A<0$ or $\mathrm{L}<0)$. In fact, the very difference between matter particles and antimatter particles is: matter particles are characterized by positive values of $\mathrm{M}$ (A or/and L) and antimatter particles are characterized by negative values of $\mathrm{M}$ (A or/and $\mathrm{L})$. As in numbers where the sum of two opposite numbers is zero, in reaction involving particles the sum of $\mathrm{A}$ or $\mathrm{L}$ of a matter particle and its antiparticle is also zero. So it is possible to create one pair of matter particle $(M=1)$ and antiparticle $(\mathrm{M}=-1)$ (for example $\mathrm{e}^{-}$and $\mathrm{e}^{+}$or $\mathrm{p}$ and $\left.\bar{p}\right)$ from a photon $\gamma$ with $(\mathrm{A}=0, \mathrm{~L}=0, \mathrm{M}=0$, $\mathrm{Q}=0$ ). We underline that matter number $\mathrm{M}$ is conserved (in fact $\mathrm{A}$ and $\mathrm{L}$ are separately conserved) but mass is not conserved: there is creation of mass from energy, in the form of a pair of matter particle and antimatter particle, particle and antiparticle having the same mass. Conversely annihilation of a pair of matter particle and antimatter particle give photons, gluons or $\mathrm{Z}$. Conservation of $\mathrm{A}$ and conservation of $\mathrm{L}$ remain valid in all reactions observed to this day, involving matter particles, antimatter particles and matter neutral particles. All these reactions are governed by electromagnetism, Strong Interaction or Weak Interaction.

\section{Definition of particle and antiparticle in the general case}

The antiparticle of a given particle is a particle with all arithmetic properties identical to those of the particle and all algebraic properties (known or yet unknown) opposite to those of the particle. By definition, a matter particle $(\mathrm{A}>0$ or $\mathrm{L}>0)$ is the particle and its antimatter particle $(\mathrm{A}<0$ or $\mathrm{L}<0)$ is the antiparticle. For neutral matter particles like mesons or messengers there is ambiguity $(\mathrm{A}=0, \mathrm{~L}=0)$. For example mesons $\pi^{-}$and $\pi^{+}$(messengers $\mathrm{W}^{-}$ and $\mathrm{W}^{+}$) form a pair of particle antiparticle, but which is particle, which is antiparticle is only 
a question of convention. It is clear that a charged particle is necessarily distinct from its antiparticle but even neutral mesons can be different from its antiparticle (for example $K 0(d \bar{s})$ and $\bar{K} 0(\bar{d} s))$. From the very definition of antiparticle, we deduce immediately that a truly neutral particle (particle identical with antiparticle) is necessarily a matter neutral particle, moreover, it cannot possess any non null algebraic property. These particles are CP invariant. We proposed methods to recognize if a neutral particle is or is not a Truly Neutral Particle (TNP) [4]

\section{9. $\alpha$ decay governed by Strong Interaction and $\beta$ decay governed by Weak Interaction}

When for example a polonium nucleus decays into a lead nucleus and an alpha particle $\left({ }^{212} \mathrm{Po} \rightarrow \alpha+{ }^{208} \mathrm{~Pb}\right.$ ), the alpha spectrum is discrete presenting peaks and we note that $\mathrm{M}$ (reduced to $\mathrm{A}$ ) and $\mathrm{Q}$ are conserved. It was thus at first thought that in beta decay, the spectrum of electron, should be of the form $n \rightarrow p+e$, and thus should be similar to alpha decay with also a spectrum presenting a peak (We remark that with hindsight, if neutron really decays via this process then it would mean conservation of $\mathrm{A}$ and $\mathrm{Q}$ but apparition of another kind of charged matter particle, thus creation of matter). In fact, experiments showed clearly that the spectrum is continuous and Pauli boldly invented a new neutral particle which should have $\mathrm{Q}=0$ and spin= $1 / 2$ in order to conserve angular momentum and $\mathrm{Q}$, these two conservation rules being considered to be true in all circumstances. This mysterious neutrino seems to play a very special role. Being a lepton without electric charge, it is only sensitive to Weak Force. The neutrino is nevertheless the fourth matter particle. It must have spin $=1 / 2$ and $\mathrm{Q}=0$ to be able to account for beta decay such as $n \rightarrow p+e+v$. And if Weak Interaction which governs neutron beta decay does respect the conservation of matter, then the third particle should be in fact an antineutrino with $\mathrm{L}=-1$. But, we must remind ourselves that the concept of baryon number and lepton number came much later. So in the 1930 s, only conservation of $\mathrm{Q}$ was explicitly required but we remark that in the case of alpha decay, conservation of baryon number even not explicitly mentioned was in fact automatically respected in the form of conservation of the number of protons and the number of neutrons (leptons are not involved).

\section{Neutron is different from antineutron, what about neutrino?}

The case of the nature of neutron was immediately solved by Racah [3] who remarked that neutron has a non null magnetic moment: this sole indisputable data was sufficient to infer univocally that neutron and antineutron are different. Consequently, neutron decays only into $\mathrm{p}+\mathrm{e}+\bar{v}$ channel and cannot decay into its conjugate channel in perfect accordance with 
the experimental fact that nobody has seen the decay of neutron into $\bar{p}+\bar{e}+v$ channel. Racah's arguments cannot be applied to the case of neutrino (magnetic moment was believed to be zero, even now it is still compatible with zero [7]). So, in 1930s there was no experimental argument against its possible Majorana character. Historically, without knowing anything else about the other properties of the neutral neutrino, Furry [8] pointed out that $\beta \beta$ decay [9] would provide an interesting way to solve its nature, in the framework of the knowledge of that time, where parity conservation was implicitly taken for granted (Beta decay is characterized as a nuclear process emitting a beta particle accompanied by a neutrino, namely an electron and an antineutrino or a positron and a neutrino, changing thus the nuclear charge $\mathrm{Z}$ by one unit and leaving mass number $\mathrm{A}$ unchanged. Double beta decay is characterized as a nuclear process emitting two beta particles changing thus the nuclear charge $\mathrm{Z}$ by two units and leaving mass number $\mathrm{A}$ unchanged. This very rare decay can occur only if the initial nucleus is less bound than the final one but more bound than the intermediate nucleus. If the neutrino is different from the antineutrino, the double beta decay will lead to the simultaneous emission of two beta particles and two neutrinos, namely two electrons and two antineutrinos or two positrons and two neutrinos. However if the neutrino and the antineutrino are the same particle the double beta decay will be without the emission of any neutrino). In effect, if neutrino and antineutrino are different, then the expected halflife of double beta decay via theoretically allowed process $\beta \beta 2 v$ decay was excessively long (about $10^{20}$ years) and its measurement was not experimentally reachable by many orders of magnitude. On the contrary, if neutrino and antineutrino are the same particle, then measurement of double beta decay (through additional process $\beta \beta 0 \mathrm{v}$ neutrinoless double beta decay allowed by the fact that it is no more necessary to distinguish neutrino and antineutrino) was accessible, this process being enhanced by a very huge phase factor of many orders of magnitude over the rate of $\beta \beta 2 v$ decay. Failures to observe double beta decay events (geochemical methods) were then logically interpreted as a proof of the difference between neutrino and antineutrino. Identity of neutrino and antineutrino was at that time considered to be necessary and sufficient condition for the occurrence of neutrinoless double beta decay.

After the overthrow of parity in Weak Interaction $[10,11]$ it was realized that $v=\nabla$ is no more sufficient to allow the occurrence of $\beta \beta 0 v$ decay, a second condition is necessary: the neutrino must have a mass different from zero (the lifetime of $\beta \beta 0 v$, if it exists, is believed now to be also linked to the mass of the neutrino and its lifetime tends to infinity when the mass tends to zero. Even if $v=v, \beta \beta 0 v$ decay is strictly forbidden if $m_{v}=0$ due to 
the impossibility to change helicity). Several review articles $[13,14]$ discussed at length the relation between $\beta \beta$ decay and the properties of the neutrino. Many theoretical models beyond the Standard Model (SM) predict the existence of $\beta \beta 0 v$ decay and thus the possibility of measuring the mass of the neutrino by this way. The first univocal evidence of $\beta \beta$ decay was observed only in 1968 [15] using geochemical method. Strictly speaking, it could be due either to $\beta \beta 2 v$ or $\beta \beta 0 v$ processes even if theory favours $\beta \beta 2 v$ process, since only lifetime was measured. The situation in 1982 was summarized by Amaldi [16]:"all double beta decay processes are likely to proceed at the more slower rate typical of the two neutrino processes (31) and it is now much more difficult to determine whether or not the no-neutrino process of Eq (32) really does occur. The signature of these processes should be looked for in the spectrum of the emitted electrons. Which of the two processes (31) (32) really takes place in nature, was a problem unsolved in 1960, and is still unsolved today". In 1987, Elliot et al [17] measured both lifetime and $\beta \beta$ spectrum in a time-projection chamber. They gave the lifetime of ${ }^{82} \mathrm{Se}$ and identified $\beta \beta$ decay to be indeed $\beta \beta 2 \nu$ process (continuous spectra without any evidence of $\beta \beta 0 v$ peak). Experiments using ${ }^{76} \mathrm{Ge}$ as source and detector proved also that $\beta \beta 2 v$ exists (continuous spectra) and have not yet detected univocally $\beta \beta 0 v$ decay event. The corresponding lower limits of lifetime are : $1.110^{25} \mathrm{y}$ [18] and $0.810^{25} \mathrm{y}$ [19]. And the most recent lower limit is $5.710^{25}$ y [20] after $24 \mathrm{kgy}$ of data with pulse shape measurement. Despite an impressive leap of the lower limit, from $1.110^{25} \mathrm{y}$ to $5.710^{25} \mathrm{y}$, due to a new method of estimating it, there is no signal of $\beta \beta 0 v$ peak. $\beta \beta 2 v$ decay has now been unambiguously detected in several nuclei (testified by continuous spectra observed in a lot of nuclei) while so far, no $\beta \beta 0 v$ decay event has been detected univocally (testified by persistent failures to observe evidence of $\beta \beta 0 \mathrm{v}$ peak in any nuclei). The present experimental status of $\beta \beta$ decay studies is still very well summarized by Fiorini [21] "No evidence, but also not even a hint, has been presented so far for the dreamed peak in the electron sum corresponding to neutrinoless double beta decay" while continuous spectra arise clearly in $\beta \beta$ decay of a lot of nuclei whenever sensitivity is sufficient. The only claimed positive evidence of $\beta \beta 0 v$ decay peak came from Klapdor-Kleingrothaus et al [22] who used a particular mathematical process but the correctness of their deduction was immediately questioned [23,24]. We remark also that, an analysis of the practically the same data, by Heidelberg Moscow collaboration [25], led only to a lower limit for the half-life. And the recent analysis of Bakalyarov et al [26] of the same collaboration concluded that "appearance of this peak does not correspond to any 
decay line in the energy range and is not connected with statistics, and cannot be considered as any evidence of neutrinoless double beta-decay". Klapdor-Kleingrothaus et al [27] responded that analysis of Bakalyarov et al [26] included some corrupt data and by excluding them they improved the confidence level of neutrinoless signal to $4.2 \sigma$. KlapdorKleingrothaus et al $[\mathbf{2 2 , 2 7 , 2 8 ]}$ claimed that their analysis correctly inferred that neutrino is identical to antineutrino with a finite mass but overlooked to mention, to discuss and to explain the apparent contradiction between Majorana character of the neutrino deduced from their interpretation and Dirac character of the neutrino necessary to explain all other clearly observed reactions where neutrino is explicitly involved.

There is a great analogy between the spectra of $\beta$ decay and $\beta \beta$ decay [29]: both spectra are continuous and no peak has been seen at maximum energy (no neutrino emission) neither in $\beta$ decay nor in $\beta \beta$ decay spectra. $\nu \nu$ plays the same role in $\beta \beta$ decay as $\nu$ in $\beta$ decay. We discussed at length the analogy and the difference between $\beta$ decay and $\beta \beta$ decay and exposed arguments leading to the conclusion that there is no peak in these two cases but for different reasons. To this day no sound experimental results (namely univocal $\beta \beta 0 v$ decay peak) require that the neutrino is a Majorana particle. This hypothesis is uniquely suggested by theories. However we must realize that even absolute absence of $\beta \beta 0 v$ process could no more infer (as before the fall of parity conservation in Weak Interaction) that neutrino is different from antineutrino. But if one can show by other means that neutrino and antineutrino are different then there should be absolute absence of $\beta \beta 0 v$ process whether the mass of the neutrino is zero or different from zero since identity of neutrino and antineutrino is a necessary condition for the existence of $\beta \beta 0 \mathrm{v}$ process. Now on one hand we do have arguments against the identity of the neutrino and the antineutrino. Neutrino is a matter particle with $\mathrm{L}=1$, does have non null weak charge (otherwise, it would not be sensitive to Weak Interaction) and we know that solar neutrinos are neutrinos while reactor neutrinos are antineutrinos which are clearly distinct by their behaviour. In effect solar neutrinos which transform ${ }^{37} \mathrm{Cl}$ into ${ }^{37} \mathrm{Ar}$ in Davis experiments [30] cannot transform proton into neutron in Reines and Cowan experiments [31]. On the contrary, reactor antineutrinos which transform proton into neutron in Reines and Cowan experiments cannot transform ${ }^{37} \mathrm{Cl}$ into ${ }^{37} \mathrm{Ar}$ in Davis experiments. We underline also that in his Nobel lecture: A half-century with solar neutrinos, Davis [31] clearly stated: "My experiment showed that the neutrino was not its own antiparticle". The inescapable conclusion is then that neutrinoless double beta decay cannot occur in nature and we could naturally explain why the "dreamed peak" has never shown up 
and will not show up whatever the sensitivity is. On the other hand we should realize that once the existence of $\beta \beta 0 \mathrm{v}$ process taken for granted (for this, neutrino should be massive and lepton number of the neutrino should be 0 as any known truly neutral particle to permit the occurrence of $\beta \beta 0 \mathrm{v}$ decay, while as any lepton, the lepton number of the neutrino should be 1 . There is internal contradiction since the lepton number of the neutrino cannot be both 0 and 1 . Indeed one has to explain this paradox if one admits that $\beta \beta 0 v$ decay takes place in nature and failures to observe it are only a question of sensitivity), there is automatically violation of $\mathrm{L}$, with $\Delta \mathrm{L}=2$. It is the reason why this strange behaviour arises only in this special case where precisely neutrinos are neither involved in the entrance channel nor in the exit channel. The statement "nuclear double beta decay which probably is the most sensitive way to look for (total) lepton number violation and probably the only way to decide the Dirac or Majorana nature of the neutrino" [27] which was true before the fall of parity in Weak Interaction is now only partially true: namely neutrino should be massive and identical to antineutrino if $\beta \beta 0 v$ peak is univocally observed in several nuclei to avoid possible misinterpretation of background peaks. We underline that we cannot decide that neutrino is a Dirac particle from the absence of $\beta \beta 0 v$ decay peak. Indeed the Dirac nature of neutron and neutrino is simply and clearly revealed by at least one non null algebraic property. Absence of the decay of neutron into $\bar{p}+\bar{e}+v$ channel and persistent failures to detect $\beta \beta 0 v$ process are in perfect accordance with this inference.

\section{Conclusion}

Conservation laws in chemical equations deal only with positive integer numbers, the number of atoms (matter) of a particular species being necessarily a positive number. To account for observed reactions in nuclear physics and particle physics we need conservation of baryon number and lepton number. These conservation laws using conservation laws in chemistry as blueprint deal with both positive (matter) and negative (antimatter) numbers. Conservation of atoms, of baryon number and lepton number can indeed be interpreted as conservation of matter (counting relation). In chemistry, conservation of atoms implies conservation of mass (positive quantity) because the variation of mass due to $Q$-value is completely negligible. In nuclear physics and particle physics, it is not the case. Conservation of matter does not mean conservation of mass. Mass and matter are not equivalent.

All known interactions of the Standard Model, namely, Electromagnetism, Strong Interaction and Weak Interaction conserve A, L and Q. Indeed Q is not a simple scalar 
represented by the algebraic value of a unidimentional vector, it is in reality intimately related to $\mathrm{BAL}=\mathrm{A}-\mathrm{L}$ and $\mathrm{TF}=\mathrm{THF}+\mathrm{TLF}[\mathbf{5}, \mathbf{6}]$

$\mathrm{Q}=\frac{B A L}{2}+\frac{T F}{2}$

$\mathrm{BAL}$ and TF are linearly independent.

Conservation of $\mathrm{Q}$ implies thus conservation of TF and conservation of BAL. Conversely, conservation of TF and BAL implies conservation of Q. Since interactions of the Standard Model conserve both $\mathrm{Q}$ and $\mathrm{M}=\mathrm{A}+\mathrm{L}$ we deduce immediately that conservation of $\mathrm{M}$ and $\mathrm{BAL}$ is equivalent to conservation of $\mathrm{A}$ and $\mathrm{L}$. Conservation of $\mathrm{Q}$ (considered as a simple scalar) is equivalent to conservation of TF. Besides in the case of Electromagnetism and Strong Interaction, each individual flavour has to be conserved while in Weak Interaction only $\mathrm{TF}$ has to be conserved. Variation of hadronic flavours can be compensated by variation of leptonic flavours so that we observe semi-leptonic reactions. Interactions of the Standard Model which conserve both matter and $\mathrm{Q}$ in microscopic level can thus not explain the observed asymmetry of our Universe which requires non conservation of matter in microscopic level. We remind ourselves that Strong Interaction was introduced to explain the stability of nuclei: there must exist a powerful force to compensate electromagnetic force which tends to cause protons to fly apart. Weak Interaction with laws of conservation different from Electromagnetism and Strong Interaction was postulated to explain beta decay. Likely, our observed material and neutral universe would be footprint of the existence of another interaction that did conserve $\mathrm{Q}$ but did not conserve matter. Conservation of BAL and TF without conservation of M (matter) would provide naturally a possible explanation of the unbalance between matter and antimatter clearly observed in our material and neutral Universe. The precise value of this excess should be considered as incidental and would correspond to initial conditions [6].

\section{References}

1) A. Pais, Progress of Theoretical Physics, 10 (1953) 457

2) E. Majorana, Nuovo Cimento, 14 (1937) 171

3) G. Racah, Nuovo Cimento, 14 (1937) 322

4) U.C. Tsan, Int.J.Mod.Phys.E13(2004) 425

5) U.C. Tsan, Int.J.Mod.Phys.E7(1998) 405

6) U.C. Tsan, Int.J.Mod.Phys.E7(1998) 747

7) Z. Daraktchieva and MUNU collaboration, Phys. Lett.B615(2005)153 
8) W.H.Furry, Phys.Rev.56 (1939), 1184

9) M.Goeppert-Mayer, Phys.Rev.48 (1935) 512

10)T.D.Lee and C.N.Yang, Phys.Rev.104 (1956) 254

11)C.S.Wu , E.Ambler, R.W.Hayward, D.D.Hoppes and R.P.Hudson, Phys.Rev.105 (1957) 1413

13) M. Moe and P. Vogel, Annu.Rev.Nucl.Part.Sci.44(1994)247 and references therein 14)A. Faessler and F. Simkovic, J.Phys.G:Nucl.Part.Phys.24(1998)2139 and references therein

15) T. Kirsten, O.A. Schaeffer, E. Norton and R. Stoenner, Phys.Rev.Lett.20(1968)1300

16) E. Amaldi, Journal de Physique, C8 (1982)261

17)S.R. Elliott, A.A. Hahn and M.K. Moe, Phys.Rev.Lett.59 (1987) 2020

18) L. Baudis et al, Phys.Lett.B.407 (1997) 219

19) C.E. Aalseth et al, Phys.Rev.C59 (1999) 2108

20) L. Baudis et al, Phys.Rev.Lett.83 (1999) 41

21) E. Fiorini, Nucl.Phys.B (Proc. Suppl.) 91(2001)262

22) H.V. Klapdor-Kleingrothaus et al, Mod.Phys.Lett A, 16(2001)2409

23)C.E.Aalseth et al, Preprint hep-ex/202018(2002)

24)Ferruccio Feruglio, Alessandro Strumia and Francesco Vissani, Preprint hep$\mathrm{ph} / 0201291(2002)$

25) H.V. Klapdor-Kleingrothaus et al , Eur.Phys. J.A 12(2001)147

26) A.M. Bakalyarov et al , hep-ex/0309016

27)H.V. Klapdor-Kleingrothaus et al , Phys.Lett.B 586(2004)198

28) H.V. Klapdor-Kleingrothaus, Nucl.Phys. B (Proc.Supple.)143(2005)229

29) U.C. Tsan, Int.J.Mod.Phys.E12(2003) 439

30) R.Davis Jr., Phys.Rev.97 (1955) 766

31) F. Reines and C.L. Cowan, Jr.,Phys.Rev. 92(1953)830

32) R.Davis Jr., Rev.Mod.Phys. 75 (2003) 985 